$\mathbb{T}$ periodica polytechnica

Civil Engineering

$54 / 1(2010) 6772$

doi: 10.3311/pp.ci.2010-1.08

web: http://www.pp.bme.hu/ci

(c) Periodica Polytechnica 2010

RESEARCH ARTICLE

\section{Analysis of the quality variances of asphalt production by Monte Carlo Simulation}

\author{
Csaba Tóth
}

Received 2009-11-10, accepted 2009-12-10

\begin{abstract}
The first part of the article discusses the control level and characteristics analysis of the production of an asphalt mixture produced by a normal asphalt mix plant. By examining the stability of production, the analysis identifies the so called process capability indices as well as the density and distribution functions of the tested parameters. With the help of these, a prediction can be made on the Operating Compliance Level (OCL) of the mixture plant provided the existing processes will remain unchanged in the future. In the second part of the article certain stochastic methods i.e. the Monte Carlo Simulation techniques are used to examine the reliability of the production process in order to provide a review on how the dynamic modulus of the asphalt mixture is influenced by the variation of the tested parameters.
\end{abstract}

\section{Keywords}

Asphalt production - Monte Carlo Simulation · dynamic modulus

\section{Csaba Tóth}

Department of Highway and Railway Engineering, BME, 1521 Budapest, Múegyetem rkp. 3., Hungary

e-mail: toth@uvt.bme.hu

\section{Introduction}

In the era of small batch production, quality control of specific product characteristics used to focus on the checking of finished products on a daily basis. The introduction of mass production brought along certain changes, as a result of which today there are well-known quality assurance systems focusing on the various phases of quality control and quality management. This trend is certainly also illustrative of the construction products including the road construction materials. This topic is especially relevant due to Hungary's accession to $\mathrm{CEN}$, which manifests itself in the harmonisation of technical regulations and Hungary's gradual adoption of the relevant European norms. This trend is particularly important and appropriate today in respect of the asphalt production.

As a result of the changed quality assurance requirements, today in Hungary asphalt mixtures can only be marketed if they bear the "CE" sign. Due to the consequences of new production control requirements and for the purpose of summarising the difficulties of transformation, it is worth taking an overview of the control level and characteristics analysis of the production, which will be dealt with in the first part of this article. The statistical parameters of mixtures and the application of the so called Monte Carlo simulation allow examining how the dynamic of the asphalt mixture is influenced by the variation of production quality. This topic is outlined in the second part of the article.

\section{Statistical quality management}

\subsection{New method of plant production control}

Probability calculi can prove that random samples taken from the production provide reliable representation of the relevant characteristics of mass products. Proof can be provided that the characteristics of the asphalt mixture have a standard distribution, on the basis of which a statistical quality assurance system can be built as it was established several decades ago by a number of countries including Hungary.

For verifying the operating compliance level of asphalt mixtures, a uniform system has been established, part of which is the EN 13108-21 Factory Production Control European Norm. This norm establishes the quality and production control re- 
quirements for the production of asphalt mixtures to be used at public roads, communal areas and airports.

Samples are taken regularly and randomly from the finished asphalt in the plant according to the relevant stipulations of the EN 12697-27 and EN 12697-28 norms. Such samples are representative of the mass production. The samples are analysed for particle-size distribution and binding agent content, and the results are categorised as compliant or non-compliant based on specified tolerance thresholds. The Operating Compliance Level (OCL) of each asphalt mixing plant will be set according to the number of non-compliant cases. An increase in the number of non-compliant cases will result in increasing the frequency of testing. If the last 32 tests results include more than 8 noncompliant cases, the plant must be shut down and an immediate and overall performance control will be required.

\subsection{Probability of non-compliant results}

The two approaches for defining the notion of quality are related to products and processes. One of the major differences between the Hungarian legacy requirements and the European requirements to be introduced is that the earlier regulations used to focus exclusively on the asphalt mixtures as finished products and tried to verify the quality of the product ("product quality"). While still relying on this aspect, the new norm goes one step forward and also intends to verify our abilities in terms of how far we are able to track the activities and how well we can cope with the current situation ("process quality").

Production processes, including asphalt production, can be regarded as stable if the tested parameters have a random variation, which is constant over the time and the variations have no recognisable reasons. If the distribution of the results is normal, the so called Gauss curve can also be drawn to provide a density function for the samples. When an adequate number of elements are available, the Gauss curve can provide a reliable description of the main characteristics of the mass. Relying on this information, predictions can be made for (i) the expected value of a specific parameter of a mixture against the prescribed value or (ii) the deviations of the mixture (i.e. the "quality variations" of production). Fig. 1 below shows the changes of particle distribution being one of the tested parameters. Considering the above graph, the production can not be regarded as regulated, because the predicted parameter value is above the prescribed value. Hence, with "p" probability, non-compliant mixture will be produced at certain periods due to exceeding the upper threshold.

The density and distribution functions structured on the basis of particle-size distribution and binding agent content data can help identify the percentage ratio of cases, when either upper or lower threshold runoff will occur.

Facilitated by the processing of a sufficient volume of reliable historical data, future behaviour patterns can be predicted e.g. the probability of data falling into a specified range. Prognosis can also be made on the Operating Compliance Level (OCL)

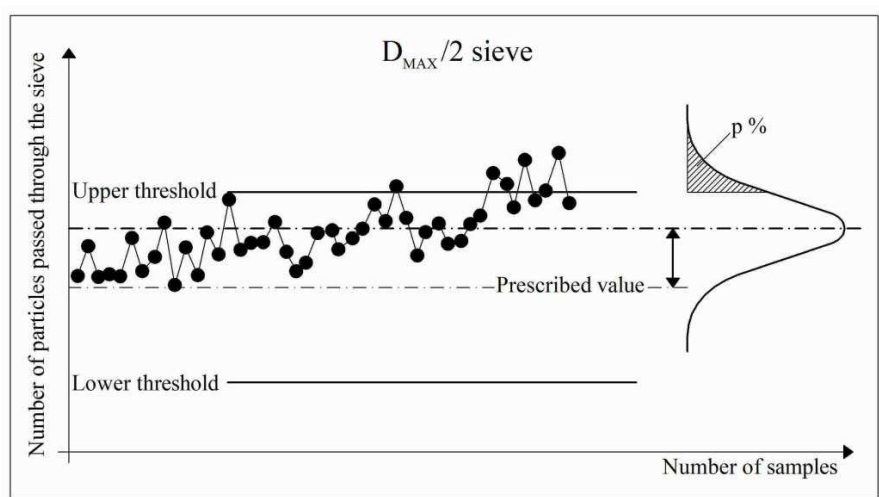

Fig. 1. $D_{\max } / 2$ particle distribution values during production

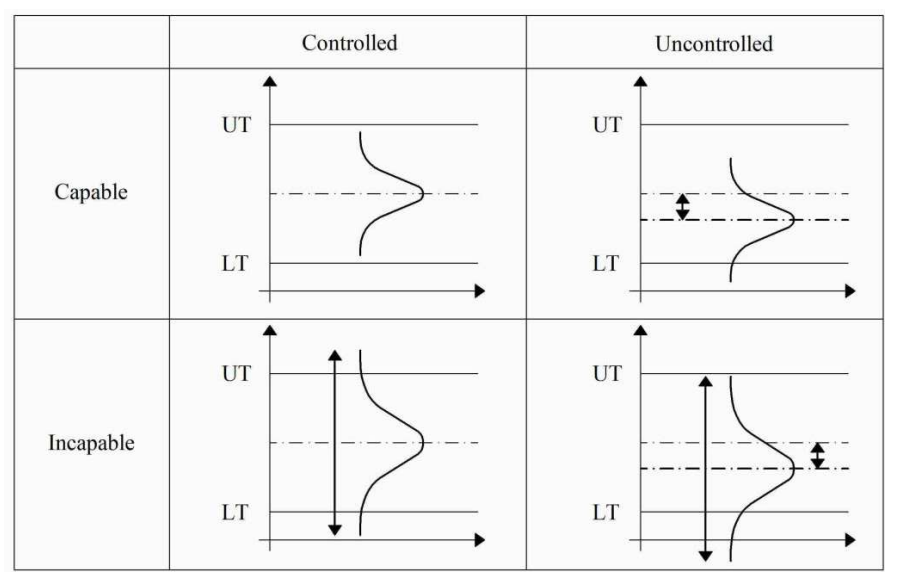

Fig. 2. Capability and control level of a process

of the mixture plant provided the existing processes will remain unchanged in the future.

\subsection{Analysis of control levels and characteristics}

In the every day practice of asphalt production it is also important to distinguish between the degree of control and the capabilities of production processes (Fig. 2). A process is deemed to be controlled if the predicted values match the prescribed values, whereas the production is regarded as uncontrolled and instable, if the performance values significantly differ from the prescribed values. Capability of a process refers to the fact that the range of a tested mixture parameter will not exceed the difference of the upper threshold (UT) and the lower threshold (LT), i.e. the operation can be maintained within the prescribed values. When an adequate number of elements are available, a statistically sound analysis of the production quality can be performed. The control level and capabilities of processes can be well described by the so called quality capability index Eq. (1).

$$
c_{p}=\frac{T R}{6 \sigma}=\frac{U T-L T}{6 \sigma} .
$$

This formula defines the ratio of tolerance range (TR) and production-specific performance range. Assuming that normal distribution prevails, the production-specific performance range will be around six times higher than the variance. If this quotient 
is above 1 , the production capability is compliant. This means that we are able to keep the production performance variance within the prescribed limits. Considering that the $c_{p}$ index is unable to recognise variations compared to the mean value, the so called corrected quality capability index is also used in the daily practice $[2,3]$ as follows:

$$
\begin{gathered}
c_{p a}=\frac{\bar{x}-L T}{3 \sigma} \\
c_{p f}=\frac{U T-\bar{x}}{3 \sigma} .
\end{gathered}
$$

Table 1 shows the quality capability indices of a mixture produced at a regular Hungarian asphalt mixture plant throughout 2007. Quality production issues are mainly important for those parameters, for which no mandatory measurements had to be carried out in Hungary before the introduction of EU norms. In such cases the quality capability of the production is noncompliant, which is an issue that needs to be dealt with. The results also show that in respect of parameters, which used to be under control even in the legacy production control system (e.g. binding agent content), the process capability do exist $\left(c_{p}>1\right.$, that is the "process is capable").

\section{Effects of the variation}

\subsection{Dynamic modulus}

In the next sections it is also worth examining what effect the inherent variances of asphalt production within the tolerance range have on the finished asphalt mixture. Referring to what has been discussed in the previous section, it can be seen that the tested particle-size distribution and binding agent content values deviate from the prescribed values to varying degrees. In view of the fact that an insufficient number of mechanical test results were available for the asphalt, there was no other choice but to apply the asphalt dynamic modulus calculation to verify the effect of production performance variations on the finished asphalt mixture.

Several prediction methods are available to define the dynamic modulus (e.g. Heukelom - Komp, Bonnaure et al., Verstraten et al., etc.) [2]. However, the disadvantage of the correlations used in these methods is that in addition to bitumen resilience, the phasic composition is also used to carry out the calculation, but for the mixture to be tested only bitumen content and particle distribution data are available. Nevertheless, the Asphalt Institute Method (Witczak et al), elaborated and widely used in the United States, may also be applied. It is described as follows:

$$
\begin{aligned}
\log E & =-0,261+0,008225 \cdot p_{200}-0,00000101 \cdot\left(p_{200}\right)^{2} \\
& +0,00196 \cdot p_{4}-0,03157 \cdot V_{a}-0,415 \cdot \frac{V_{\mathrm{b}, \mathrm{eff}}}{\left(V_{\mathrm{b}, \mathrm{eff}}+V_{a}\right)} \\
& +\frac{1,87+0,002808 \cdot p_{4}+0,0000404 \cdot p_{38}}{1+e^{(-0,716 \cdot \log (f)-0,7425 \cdot \log (\eta))}} \\
& +\frac{-0,0001786 \cdot\left(p_{38}\right)^{2}+0,0164 \cdot p_{34}}{1+e^{(-0,716 \cdot \log (f)-0,7425 \cdot \log (\eta))}} .
\end{aligned}
$$

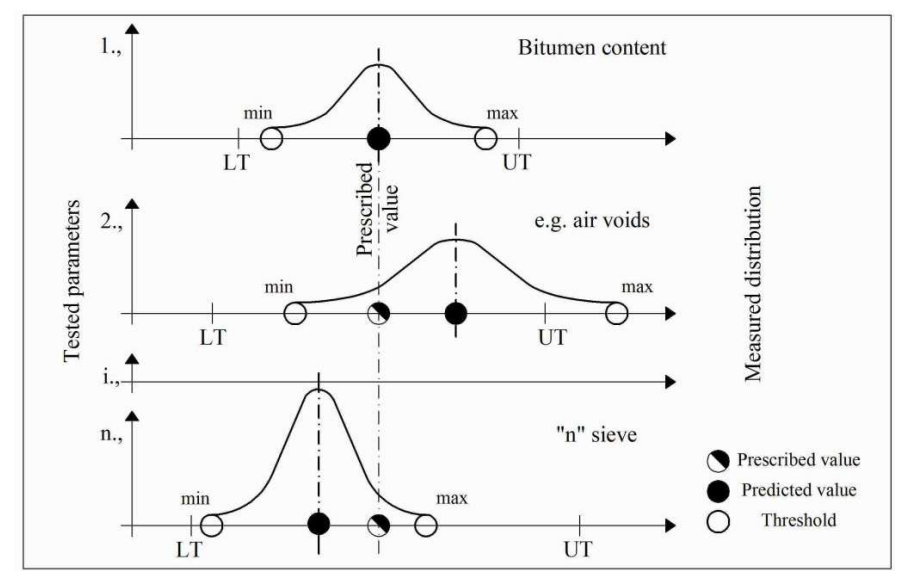

Fig. 3. Schematic description of the stochastic approach

Where the variables represent:

E Asphalt Mix Dynamic Modulus, in $10^{5} \mathrm{psi}$

$\eta$ Bitumen viscosity in $10^{6}$ poise (at any temperature, defree of aging)

\section{$f$ Load frequency in $\mathrm{Hz}$}

$V_{a} \%$ air voids in the mix, by volume

$V_{\mathrm{b}, \mathrm{eff}} \%$ effective bitumen content, by volume

$p_{34} \%$ retained on the $3 / 4$ inch sieve, by total aggregate (cumulative)

$p_{38} \%$ retained on the $3 / 8$ inch sieve, by total aggregate (cumulative)

$p_{4} \%$ retained on the No. 4 sieve, by total aggregate (cumulative)

$p_{200} \%$ retained on the No. 200 sieve, by total aggregate (cumulative).

In addition to the already tested bitumen content and particle distribution data, the above formula also relies on bitumen viscosity and air-voids input data. This correlation is based on 205 mixture tests carried out over 30 years, 171 and 34 of which used unmodified and modified binding material respectively.

To justify the reliability of production, we have density functions for historical particle distribution and bitumen content data available, but this is exactly the fact that makes the analysis complicated (see Fig. 3). It is evident that the use of prescribed data in the Witczak formula (2) would provide largely inaccurate results, because the real production input data is significantly different form the prescribed data. The modulus provides more accurate estimations, if we use the predicted values of the available parameters. However, in this case we are unable to take the effect of variations into account, which prevents us from realising the effect of raw material variations on the variance of mixture modulus. The solution of this conflict is to shift from the above mentioned deterministic routine to the stochastic approach. 
Tab. 1. Quality capability indices of tested mixtures

\begin{tabular}{cccccccc}
\hline \multirow{2}{*}{ Quality capability index } & \multicolumn{4}{c}{ Particle distribution parameters } & \multicolumn{2}{c}{ Binding agent content } \\
\cline { 2 - 8 } & & $D_{\max }$ & $D_{\max } / 2$ & $2 \mathrm{~mm}$ & Fine sieve & $0,063 \mathrm{~mm}$ & $B \%$ \\
\hline \multirow{2}{*}{ (Number of samples = 36) } & $c_{p}$ & 1.87 & $\mathbf{0 . 6 3}$ & $\mathbf{0 . 8 0}$ & 1.56 & $\mathbf{0 . 9 6}$ & 1.15 \\
\cline { 2 - 8 } & $c_{p f}$ & 1.37 & $\mathbf{0 . 7 3}$ & $\mathbf{0 . 9 4}$ & 1.51 & $\mathbf{0 . 2 7}$ & 1.07 \\
\cline { 2 - 8 } & $c_{p a}$ & 2.36 & $\mathbf{0 . 5 3}$ & $\mathbf{0 . 6 6}$ & 1.61 & 1.66 & 1.22 \\
\hline
\end{tabular}

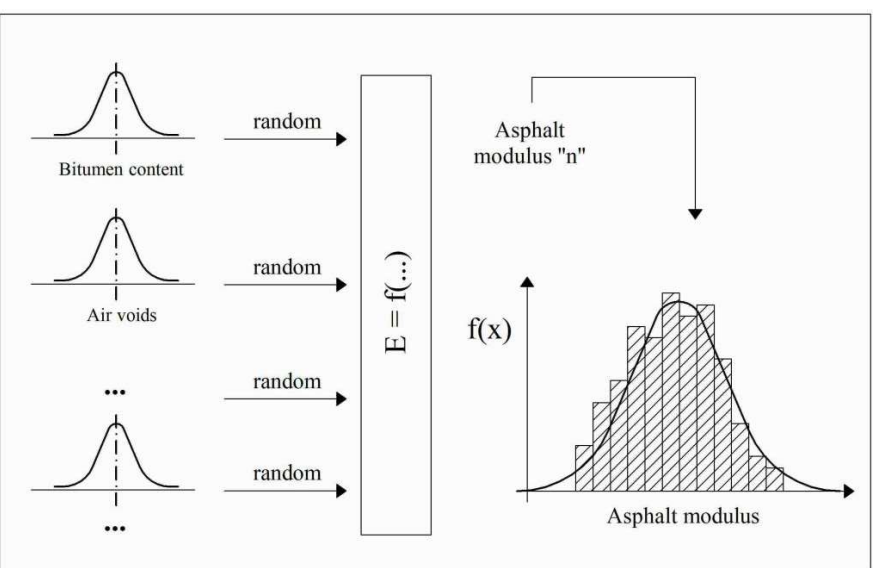

Fig. 4. Schematic drawing for the Monte Carlo method

\subsection{Monte Carlo Simulation}

Problems like the one presented here can not be managed through exact mathematic methods due to the complexity of the stochastic processes. And, the solution can not be defined in a closed form either. This is the point, when the so called simulation techniques may be applied, one of which is the Monte Carlo method. The origin of this method dates back to the " 40 s and is associated with János Neumann. The Monte Carlo Simulation is one of the most frequently used practical tool for economic and mathematical analyses, which can also be used under Excel, if facilitated by a special module. In essence, with this method we define the real distribution of all input data, from which we take random samples to select the necessary input data in order to identify the modulus of the asphalt mixture. Subsequent to a sufficiently large number of runs, a frequency histogram for the modulus of the asphalt mixture can be created, which helps estimate and analyse the distribution of the modulus (see Fig. 4). Having run the simulation with the real particle distribution and bitumen content data of the tested asphalt mixture, one can create a simulated density function. The calculated figures were as follows: predicted value: $9,808 \mathrm{MPa}$; variance of the modulus: $661 \mathrm{MPa}$; range: 7,617 - 12,381 MPa. (Fig. 5) As part of the analysis it can be examined what effect each parameter has on the estimated modulus of the finished asphalt mixture. The sensitivity diagram is shown in Fig. 6, which describes that the modulus of the asphalt mixture primarily depends on the air void content and the quantity that have passed through the $16 \mathrm{~mm}$ and $4 \mathrm{~mm}$ sieves. According to the Witczak correlation, the particle distribution below 0.063 holds very little significance. The ac- curacy of these types of forecasts is certainly debatable, where the reliability of the calculation stems from the following two key reasons:

\section{- "Inaccuracy of the applied empirical models / formulas"}

This is not surprising, because we know and use the van der Poel diagram and correlations such as the one from Verstraten or Witczak. And we understand that they are mere empiric correlations with varying success to reflect the reality. In fact, science needs to improve to be able to increase accuracy. We are left with using the above methods until better, more accurate and more reliable correlations are developed.

- "Reliability of the input data" It is crucial to use statistical methods to unfold the characteristics of all input data. Adjusting the variance of base input data can increase or decrease the level of reliability.

This latter reason signifies the importance of statistical quality management, since the more reliable our data, the more accurate forecast we will be able to create.

\section{3 "The asphalt mixture will be put to the test in the pave-} ment!"

Having seen previously the natural inherent variations of asphalt production and having accepted the simulation results showing variations of the produced asphalt mixture in the range of 7,617 - 12,381 MPa due to the fluctuation of particle distribution and bitumen feeding, these facts raise the question of what implications they have for the dimensioning of pavements. The principles of pavement dimensioning are the same as those of other disciplines, namely the strength $(\mathrm{S})$ of the structure (in this case the pavement) should be higher than the load (L) (in this case the traffic). In an ideal world these two parameters could be accurately defined on the basis of deterministic principles. It is enough, however, to consider the process of defining the traffic load or the temperature dependency of pavement characteristics or the inherent uncertainties of the pavement's geometrical sizes to be able to see that these are all stochastic values. Moreover, due to cost efficiency considerations, it is not possible to ensure that each of the "probable values" for strength will be higher than each of the corresponding "probable values" for load. It is inevitable to accept, with a certain "p" probability, the risk of premature fatigue. Therefore, it is worth considering in this context whether the variance of a pavement layer's modulus can significantly increase or decrease the inherent "p" risk 
Fig. 5. Simulated density function of the dynamic modulus values

Fig. 6. Sensitivity analysis of the asphalt mixture

Fig. 7. Principles of pavement dimensioning
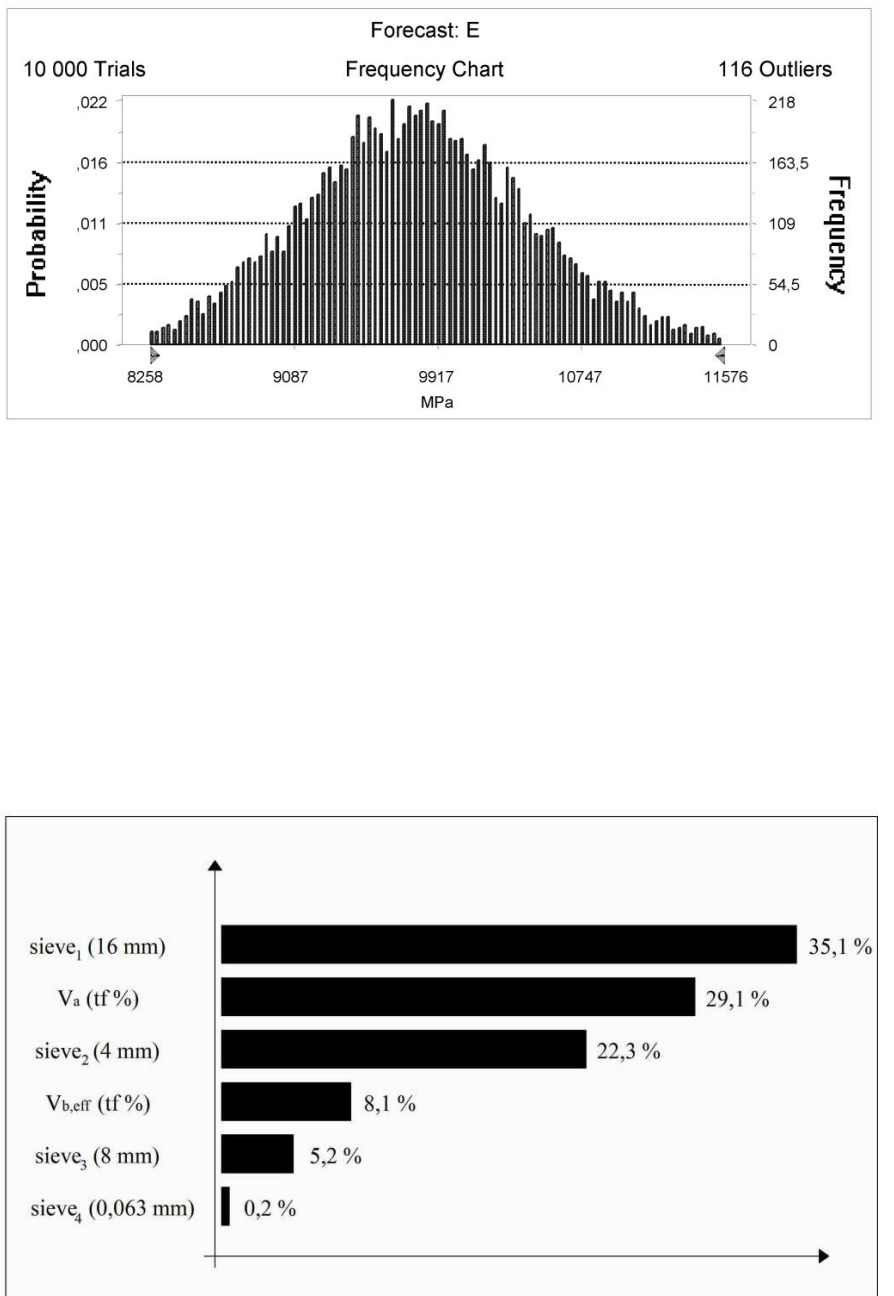

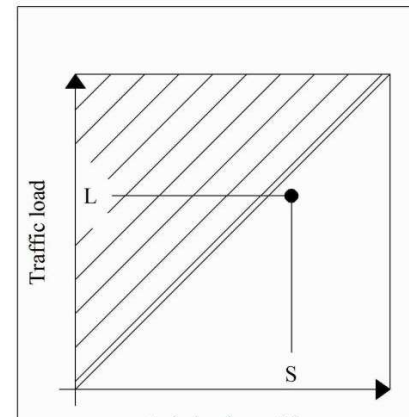

Axle load repetition

a.)

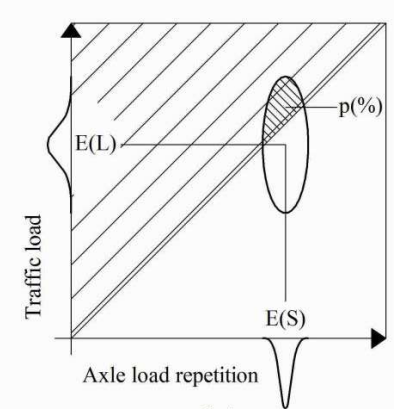

b.) 
of dimensioning.

With no consideration for the detailed discussion of stochastic phenomena, it is verifiable that if the expected value of the produced asphalt modulus is the same as the value prescribed for the mixture (i.e. the value that has been taken into account for the purpose of dimensioning), based on our current dimensioning practice the variances of the mixture has no tangible influence on dimensioning and the expected strength $(\mathrm{S})$ value. If the variance of the mixture's modulus is higher than the inherent significant variance of dimensioning, it slightly increases the level of " $p$ " risk. Consequently, if this variance is lower, it slightly decreases the level of " $p$ " risk.

Although the current Hungarian pavement planning practice is still based on a deterministic approach, it is predictable that the stochastic approach will emerge and gain phenomenon also in this field [1].

\section{Summary}

The previous sections aimed at supporting the idea that despite the performance variances, throughout the asphalt production - and the production of any other product - the characteristics of the asphalt mixture can comply with the contractual requirements in accordance with the CEN norms. Having examined how the variances of a single mixture affect the dimensioning of pavements, proof can be provided that these variances are annulled due to empirical characteristics of the dimensioning methods and the uncertainties caused by several other input parameters. These variances will gain significance only if there is a major gap between the predicted value of the mixture's modulus and the prescribed value of the same. However, this gap will gain importance only if there is a large deviation for the modulus, which is unlikely to occur if the requirements of plant production control are complied with.

This statement, on the other hand, indirectly highlights the importance of analytic pavement dimensioning, since this is a built-in gap for the traditional mixtures and the ones with high modulus. Nevertheless, these gaps can and should be taken into account. Therefore, this is not the challenge of the distant future but that of today to update the current Hungarian pavement dimensioning practice and then subsequently harmonise the quality assurance system with the technical regulatory system.

\section{References}

1 Fi I., Pethö L., Calculation of the equivalent temperature of pavement structures, Periodica Polytechnica Civil Engineering 52 (2008), no. 2, 91-96, DOI 10.3311/pp.ci.2008-2.05.

2 Pethö L, Influence of temperature distribution on the fatigue and mix design of asphalt pavement structures, Budapest University of Technology and Economics, H-1111 Budapest, Múegyetem rkp. 3, Hungary, 2008.

3 Timm David, Newcomb David, Perpetual pavement design for flexible pavements in the US, International Journal of Pavement Engineering 7 (2006), no. 2, 111-119, DOI 10.1080/10298430600619182. 